\title{
Lattice study of the Boer-Mulders transverse momentum distribution in the pion
}

\author{
M. Engelhardt ${ }^{* a \dagger}$, B. Musch ${ }^{b}$, P. Hägler ${ }^{c}$, J. Negele ${ }^{d}$, and A. Schäfer ${ }^{e}$ \\ ${ }^{a}$ Department of Physics, New Mexico State University, Las Cruces, NM 88003, USA \\ ${ }^{b}$ Theory Center, Jefferson National Laboratory, Newport News, VA 23606, USA \\ ${ }^{c}$ Institut für Kernphysik, Johannes Gutenberg-Universität Mainz, 55128 Mainz, Germany \\ ${ }^{d}$ Center for Theoretical Physics, Massachusetts Institute of Technology, Cambridge, MA 02139, \\ USA \\ ${ }^{e}$ Institut für Theoretische Physik, Universität Regensburg, 93040 Regensburg, Germany \\ ${ }^{\dagger} E$-mail: engel@ nmsu. edu
}

The Boer-Mulders transverse momentum-dependent parton distribution (TMD) characterizes polarized quark transverse momentum in an unpolarized hadron. Techniques previously developed for lattice calculations of nucleon TMDs are applied to the pion. These techniques are based on the evaluation of matrix elements of quark bilocal operators containing a staple-shaped Wilson connection. Results for the Boer-Mulders transverse momentum shift in the pion, obtained at a pion mass of $m_{\pi}=518 \mathrm{MeV}$, are presented and compared to corresponding results in the nucleon.

31st International Symposium on Lattice Field Theory - LATTICE 2013

July 29 - August 3, 2013

Mainz, Germany

${ }^{*}$ Speaker. 


\section{Introduction}

Transverse momentum-dependent parton distribution functions [1] (TMDs) encode information about the distribution of transverse (as well as longitudinal) momentum among partons in a hadron, as extracted from physical processes such as semi-inclusive deep inelastic scattering (SIDIS) or the Drell-Yan (DY) process. Isolating hadron structure information in terms of distribution functions of this type requires a factorization framework which allows one to disentangle that information from other components of the process at hand. For processes containing multiple hadrons in both initial and final states, factorization breaking contributions may exist [2], the quantitative importance of which is yet unclear. For SIDIS and DY, on the other hand, factorization does not seem to face serious obstacles, one possible approach having been advanced in [3-5], although potential issues regarding the analysis of azimuthal asymmetries have recently been noted [6].

A schematic illustration of the principal elements involved in a description of SIDIS is given by Fig. 1; they include the hard, perturbative vertex, a TMD encoding the structure of the nucleon, and a fragmentation function describing the hadronization of the struck quark. It is important to note that factorization does not necessarily imply that TMDs can be defined completely independent of the process in which they are embedded. In particular, as also indicated in Fig. 1, final-state gluon exchanges between the struck quark and the hadron remnant decisively influence the description of SIDIS (in the DY process, initial-state interactions play an analogous role). Including these finalstate interactions also modifies the momentum distributions encoded in the TMDs; the manner in which they formally enter the theoretical definition of TMDs will be elucidated further below.

These final state effects are important in that they break time-reversal invariance and thus generate nontrivial T-odd TMDs, leading to corresponding angular asymmetries in experimental cross sections. Signatures of this kind have indeed been observed experimentally [7]. Nevertheless, despite the process dependence introduced by accounting for such effects, a "modified universality" across different process types can be retained in a suitable factorization scheme. Specifically, SIDIS and DY can be parametrized by the same TMDs, up to a change in sign in T-odd TMDs [8].

The project presented here focuses on connecting the phenomenological definition of TMDs, introduced below, to a concrete lattice QCD calculational scheme, on the basis of which selected TMD observables are evaluated from first principles. In particular, exploratory results for the T-odd Boer-Mulders transverse momentum shift in the pion are given, thus expanding on the initial study of nucleon TMDs reported in [9].

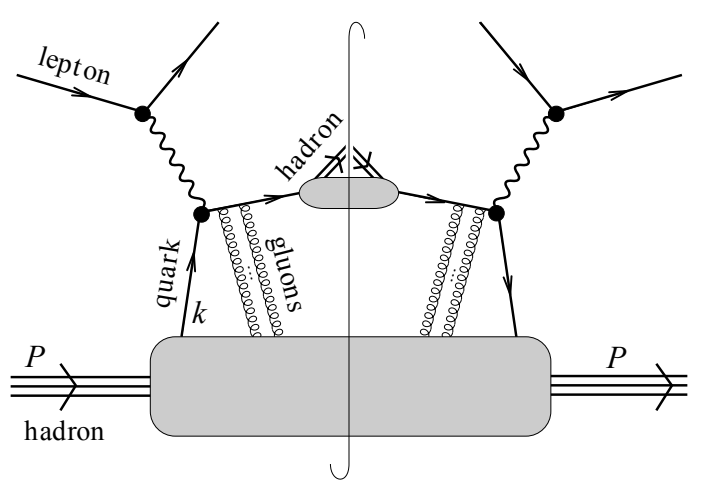

Figure 1: Illustration of the elements of SIDIS factorization. The lower shaded bubble represents the structure parametrized by TMDs. 


\section{Definition of TMD observables}

The fundamental correlator defining quark TMDs is of the form

$$
\Phi^{[\Gamma]}\left(x, k_{T}, P, S, \ldots\right)=\left.\int \frac{d^{2} b_{T}}{(2 \pi)^{2}} \int \frac{d(b \cdot P)}{(2 \pi) P^{+}} \exp \left(i x(b \cdot P)-i b_{T} \cdot k_{T}\right) \frac{\widetilde{\Phi}_{\text {unsubtr. }}^{[\Gamma]}(b, P, S, \ldots)}{\widetilde{\mathscr{S}}\left(b^{2}, \ldots\right)}\right|_{b^{+}=0}
$$

with

$$
\widetilde{\Phi}_{\text {unsubtr. }}^{[\Gamma]}(b, P, S, \ldots) \equiv \frac{1}{2}\langle P, S|\bar{q}(0) \Gamma \mathscr{U}[0, \eta v, \eta v+b, b] q(b)| P, S\rangle
$$

The standard phenomenological description employs a Lorentz frame in which the hadron of mass $m_{h}$ propagates with a large momentum in 3-direction, $P^{+} \equiv\left(P^{0}+P^{3}\right) / \sqrt{2} \gg m_{h}$; then, the quark momentum components scale such that the correlator (2.1) and TMDs derived from it are principally functions of the quark longitudinal momentum fraction $x=k^{+} / P^{+}$and the quark transverse momentum vector $k_{T}$, with the dependence on the component $k^{-} \equiv\left(k^{0}-k^{3}\right) / \sqrt{2} \ll m_{h}$ becoming ignorable in this limit. Correspondingly, (2.1) is regarded as having been integrated over $k^{-}$; thus, in the Fourier transform, the conjugate component $b^{+}$is set to zero, as written. The hadron momentum and spin are denoted by $P$ and $S$, and $\Gamma$ stands for an arbitrary $\gamma$-matrix structure. The ellipsis in $\Phi^{[\Gamma]}\left(x, k_{T}, P, S, \ldots\right)$ indicates that the correlator will depend on various further parameters, related, e.g., to regularization, specified below as needed. Heuristically, one can view the Fouriertransformed bilocal quark bilinear operator as counting quarks of momentum $k$ in the hadron state, with $\Gamma$ controlling the specific spinor components involved. However, gauge invariance additionally enforces the introduction of a gauge connection $\mathscr{U}$, the precise path of which will be specified presently. The presence of $\mathscr{U}$ introduces divergences additional to the field renormalizations of the quark operators (this is indicated by the subscript "unsubtr."); these divergences accordingly must be compensated by the additional "soft factor" $\widetilde{\mathscr{S}}$. Here, $\widetilde{\mathscr{S}}$ will not need to be specified in detail, since only appropriate ratios in which the soft factors cancel will ultimately be considered.

The gauge link structure $\mathscr{U}$ plays a natural role in the correlator (2.2), providing a vehicle for incorporating the final state gluon exchanges between struck quark and hadron remnant discussed in connection with Fig. 1. An effective resummation of these interactions yields a Wilson line which approximately follows the trajectory of the struck quark, close to the light cone. The correlator (2.2), representing the squared amplitude of the physical process, thus has parallel Wilson lines attached to both of the quark operators, extending to large distances along a direction $v$ close to the light cone; at the far end, these lines are connected by a Wilson line in the $b$ direction to maintain gauge invariance. The result is the staple-shaped connection $\mathscr{U}[0, \eta v, \eta v+b, b]$, where the path links the positions in the argument of $\mathscr{U}$ with straight line segments, and $\eta$ parametrizes the length of the staple. Formally, it is the introduction of the additional vector $v$ which breaks the symmetry under time reversal and thus generates T-odd TMDs.

At first sight, the most convenient choice for the staple direction $v$ would seem to be a light-like vector. However, beyond tree level, this introduces rapidity divergences which require regularization. One advantageous way to accomplish this is to take $v$ slightly off the light cone into the space-like region [3,4], with perturbative evolution equations governing the approach to the light cone [5]. This scheme features the modified universality alluded to further above; the SIDIS and DY processes are connected by inversion of $v$, inducing the proper sign change in T-odd TMDs. A 
scheme in which $v$ (along with the quark operator separation $b$ ) is generically space-like is also attractive as a starting point for the development of the lattice QCD calculation, as will be discussed below. A useful parameter characterizing how close $v$ is to the light cone is the Collins-Soper evolution parameter $\hat{\zeta}=v \cdot P /(|v||P|)$, in terms of which the light cone is approached for $\hat{\zeta} \rightarrow \infty$.

Decomposing the correlator $\Phi^{[\Gamma]}\left(x, k_{T}, P, S, \ldots\right)$ into the relevant Lorentz structures yields the TMDs as coefficient functions. Whereas in the nucleon case, this leads to eight distinct leadingtwist TMDs, in the simpler $S=0$ pion case investigated here, only two leading-twist TMDs remain, namely, the unpolarized TMD $f_{1}$ and the T-odd Boer-Mulders TMD $h_{1}^{\perp}$, given, respectively, by

$$
\Phi^{\left[\gamma^{+}\right]}=f_{1} \quad \Phi^{\left[i \sigma^{i+} \gamma^{5}\right]}=\frac{\varepsilon_{i j} k_{j}}{m_{\pi}} h_{1}^{\perp}
$$

The latter characterizes the distribution of transversely polarized quarks in the (unpolarized) pion.

On the other hand, also the position space correlator $\widetilde{\Phi}_{\text {unsubtr. }}^{[\Gamma]}$, cf. (2.2), which represents the quantity amenable to lattice evaluation, can be decomposed analogously in terms of invariant amplitudes $\widetilde{A}_{i B}$. Again, for $S=0$, only two amplitudes from the full set obtained for non-zero spin [9] remain,

$$
\frac{1}{2 P^{+}} \widetilde{\Phi}_{\text {unsubtr. }}^{\left[\gamma^{+}\right]}=\widetilde{A}_{2 B} \quad \frac{1}{2 P^{+}} \widetilde{\Phi}_{\text {unsubtr. }}^{\left[i \sigma^{i+} \gamma^{5}\right]}=i m_{\pi} \varepsilon_{i j} b_{j} \widetilde{A}_{4 B}
$$

These amplitudes are useful in that they can be evaluated in any desired Lorentz frame, including one particularly suited for the lattice calculation. In view of (2.3), they are clearly closely related to Fourier-transformed TMDs. Performing the corresponding algebra, and specializing, for the purposes of the present investigation, to the lowest $x$-moment by choosing $b \cdot P=0$, one has

$$
\begin{aligned}
\tilde{f}_{1}^{[1](0)}\left(b_{T}^{2}, \hat{\zeta}, \ldots, \eta v \cdot P\right) & =2 \widetilde{A}_{2 B}\left(-b_{T}^{2}, b \cdot P=0, \hat{\zeta}, \eta v \cdot P\right) / \widetilde{\mathscr{S}}\left(b^{2}, \ldots\right) \\
\tilde{h}_{1}^{\perp[1](1)}\left(b_{T}^{2}, \hat{\zeta}, \ldots, \eta v \cdot P\right) & =2 \widetilde{A}_{4 B}\left(-b_{T}^{2}, b \cdot P=0, \hat{\zeta}, \eta v \cdot P\right) / \widetilde{\mathscr{S}}\left(b^{2}, \ldots\right)
\end{aligned}
$$

where the generic Fourier-transformed TMD is defined as

$$
\tilde{f}^{[1](n)}\left(b_{T}^{2}, \ldots\right)=n !\left(-\frac{2}{m_{N}^{2}} \partial_{b_{T}^{2}}\right)^{n} \int_{-1}^{1} d x \int d^{2} k_{T} e^{i b_{T} \cdot k_{T}} f\left(x, k_{T}^{2}, \ldots\right)
$$

The $b_{T} \rightarrow 0$ limit formally yields $k_{T}$-moments of TMDs. However, this limit contains additional singularities, which one can view as being regulated by a finite $b_{T}$. Here, results will only be given at finite $b_{T}$. It is important to note the presence of the soft factors $\widetilde{\mathscr{S}}$ on the right-hand sides of (2.5) and (2.6). Absent an evaluation of these soft factors, which themselves depend on $b^{2}$, one cannot directly Fourier-transform the amplitudes $\widetilde{A}_{i B}$ extracted from a lattice calculation back to momentum space to obtain the original TMDs defined in (2.3). On the other hand, one can construct an observable in which the soft factors cancel by normalizing the (Fourier-transformed) Boer-Mulders function (2.6) by the unpolarized TMD (2.5), which essentially counts the number of valence quarks. Thus, one defines the "generalized Boer-Mulders shift"

$$
\left\langle k_{y}\right\rangle_{U T}\left(b_{T}^{2}, \ldots\right) \equiv m_{\pi} \frac{\tilde{h}_{1}^{\perp[1](1)}\left(b_{T}^{2}, \ldots\right)}{\tilde{f}_{1}^{[1](0)}\left(b_{T}^{2}, \ldots\right)}=m_{\pi} \frac{\widetilde{A}_{4 B}\left(-b_{T}^{2}, 0, \hat{\zeta}, \eta v \cdot P\right)}{\widetilde{A}_{2 B}\left(-b_{T}^{2}, 0, \hat{\zeta}, \eta v \cdot P\right)}
$$

which is the regularized, finite- $b_{T}$ generalization of the "Boer-Mulders shift"

$$
m_{\pi} \frac{\tilde{h}_{1}^{\perp[1](1)}(0, \ldots)}{\tilde{f}_{1}^{[1](0)}(0, \ldots)}=\left.\frac{\int d x \int d^{2} k_{T} k_{y} \Phi^{\left[\gamma^{+}+s^{j} i \sigma^{j+} \gamma^{5}\right]}\left(x, k_{T}, P, \ldots\right)}{\int d x \int d^{2} k_{T} \Phi^{\left[\gamma^{+}+s^{j} i \sigma^{j+} \gamma^{5}\right]}\left(x, k_{T}, P, \ldots\right)}\right|_{s_{T}=(1,0)}
$$


which, in view of the right-hand side, formally represents the average transverse momentum of quarks polarized in the transverse (" $T$ ") direction orthogonal to said momentum in an unpolarized (" $U$ ") pion, normalized to the corresponding number of valence quarks. In the interpretation of (2.9), it should be noted that the numerator sums over the contributions from quarks and antiquarks, whereas the denominator contains the difference between quark and antiquark contributions, thus giving the number of valence quarks. Note furthermore that ratios of the type (2.8) also cancel $\Gamma$ independent multiplicative field renormalization constants attached to the quark operators in (2.2) at finite physical separation $b$.

\section{Lattice evaluation and results}

The formal framework laid out above provides all the necessary elements for a lattice QCD evaluation of the generalized shift (2.8). The path towards this observable proceeds via the calculation of pion matrix elements of the type (2.2), yielding the relevant invariant amplitudes $\widetilde{A}_{i B}$ via (2.4). This requires a setting in which the four-vectors $b$ and $v$ are generically space-like: The standard scheme for obtaining matrix elements such as (2.2) operates with (ratios of) Euclidean space-time correlators, in which evolution in Euclidean time serves to suppress pion excited states between, on the one hand, pion source and sink and, on the other hand, the operator inserted at an intermediate Euclidean time. In this scheme, only matrix elements of operators defined at a single Minkowski time are straightforward to evaluate; finite Minkowski time separations in the operator cannot be directly accomodated on the Euclidean lattice. Only if all parts of the matrix element under consideration can be evolved in time to a single instant does rotation between Euclidean and Minkowski space become trivial. Consequently, lattice evaluation of the matrix element (2.2) requires generically space-like $b$ and $v$, since only then is there no obstacle to boosting the problem to a Lorentz frame in which $b$ and $v$ are purely spatial, and calculating $\widetilde{\Phi}_{\text {unsubtr. }}^{[\Gamma]}$ in that frame. The results extracted for the invariant amplitudes $\widetilde{A}_{i B}$ are then immediately valid also in the original frame in which (2.2) was initially defined, thus completing the determination of the shift (2.8).

Since, in a numerical lattice calculation, the staple extent $\eta$ necessarily remains finite, two extrapolations must be performed from the generated data, namely, $\eta \rightarrow \infty$, as well as the extrapolation of the staple direction toward the light cone, $\hat{\zeta} \rightarrow \infty$. In a previous investigation of nucleon TMDs [9], the former extrapolation was seen to be under control for a range of parameters, whereas the latter presented a formidable challenge. The main limitation in this respect is the set of hadron momenta $P$ accessible with sufficient statistical accuracy. One of the main motivations for the present pion study was to achieve progress with respect to the large- $\hat{\zeta}$ limit. The pion, by virtue of its lower mass compared to the nucleon (note that the hadron mass enters the denominator of $\hat{\zeta}$ ), allows one to access higher $\hat{\zeta}$; also, being spinless, it allows one to obtain better statistics for the TMD matrix element (2.2) by averaging over spatial rotations of the operator under consideration. The calculations presented in the following were performed using a MILC 2+1-flavor gauge ensemble [10] on $20^{3} \times 64$ lattices with a spacing of $a=0.12 \mathrm{fm}$, corresponding to pion mass $m_{\pi}=518 \mathrm{MeV}$. The largest $\hat{\zeta}$ value reached is $\hat{\zeta}=2.03$. Disconnected contributions to the matrix elements (2.2) were not evaluated.

Fig. 2 shows a typical result for the generalized Boer-Mulders shift (2.8) for $u$-quarks at given $\left|b_{T}\right|$ and $\hat{\zeta}$ as a function of the staple extent, comparing the result for a $\pi^{+}$meson with the case of 

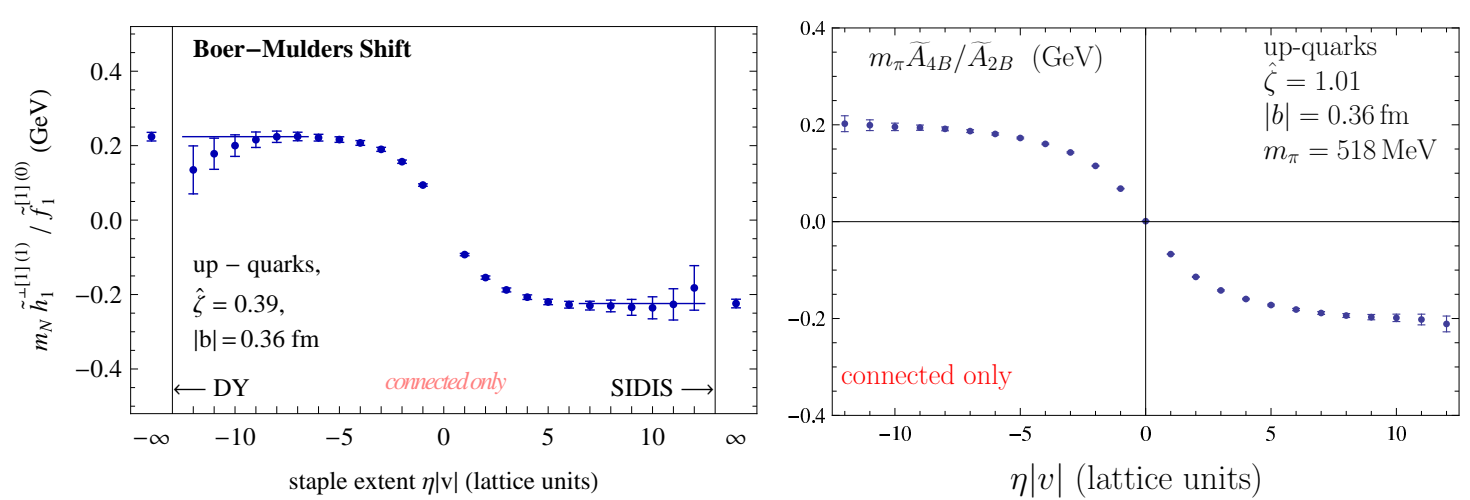

Figure 2: Generalized Boer-Mulders shift as a function of staple extent for $u$-quarks in a proton (left) and a $\pi^{+}$meson (right). Data are obtained in the same, $m_{\pi}=518 \mathrm{MeV}$, gauge ensemble at identical $\left|b_{T}\right|$ and $m_{h} \hat{\zeta}$.
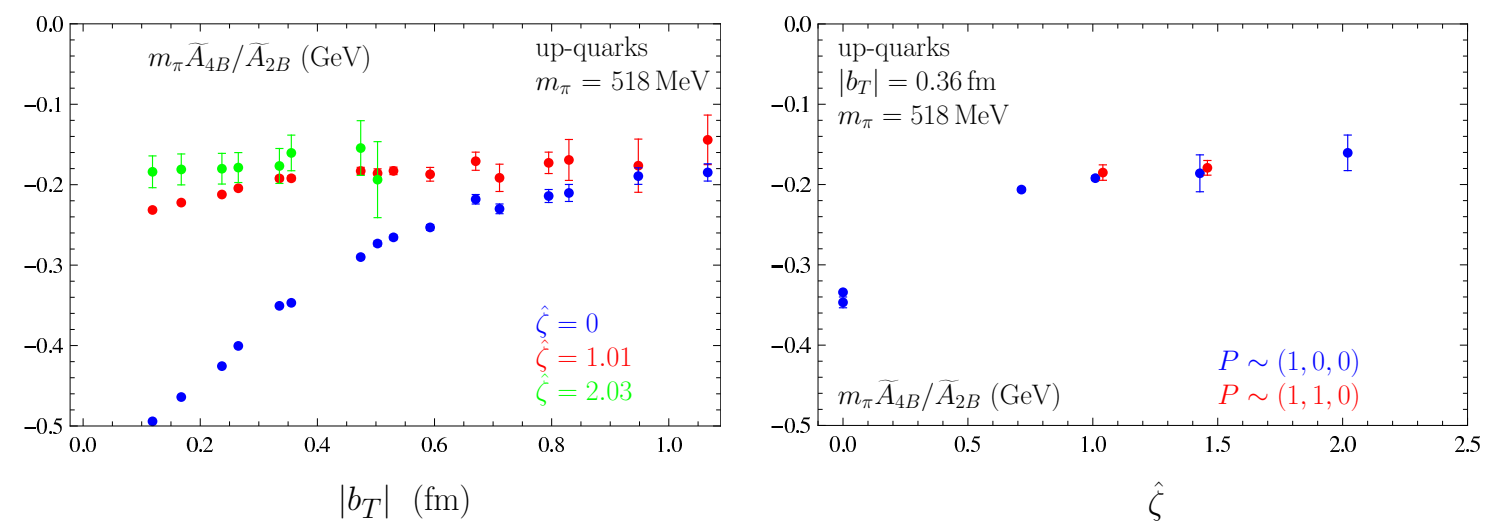

Figure 3: Generalized Boer-Mulders shift in the $\eta \rightarrow \infty$ SIDIS limit as a function of $\left|b_{T}\right|$ (left) and $\hat{\zeta}$ (right). In the left panel, the data in the region below $\left|b_{T}\right| \approx 0.25 \mathrm{fm}$ may be significantly affected by finite lattice cutoff effects. In the right panel, the congruence of the data obtained for $P$ in different directions exhibits the good rotational properties of the calculation.

a proton studied previously in [9]. The T-odd behavior of this observable is evident, with $\eta \rightarrow \infty$ corresponding to the SIDIS limit, whereas $\eta \rightarrow-\infty$ yields the DY limit. The data level off to approach clearly identifiable, stable plateaux as the staple length grows. Note that the two sets of data correspond to identical hadron momentum $P$, and the corresponding $\hat{\zeta}$ values differ only because of the hadron mass in the denominator of $\hat{\zeta}$; i.e., $m_{h} \hat{\zeta}$ is the same in the two cases. In this particular juxtaposition, the Boer-Mulders shifts are quantitatively very close to one another, in accordance with a suggestion put forward in [11]. Note, however, that this observation is special to $u$-quarks; the $d$-quark Boer-Mulders shift in the proton is significantly stronger than the $u$-quark shift, whereas the $u$-quark and $\bar{d}$-quark shifts in the $\pi^{+}$are, of course, identical.

Fig. 3 focuses on the data in the SIDIS limit. The left panel shows the dependence on $\left|b_{T}\right|$ for three different values of the Collins-Soper evolution parameter $\hat{\zeta}$; the dependence flattens as $\hat{\zeta}$ is increased. The right panel exhibits the $\hat{\zeta}$-dependence at a representative $\left|b_{T}\right|$. A considerably smaller statistical uncertainty is achieved compared to the nucleon case studied previously [9], affording a first glimpse of asymptotic behavior in $\hat{\zeta}$. The data suggest a rather early onset of the large $-\hat{\zeta}$ 
regime, which, if substantiated further in calculations closer to the physical point, would be very favorable for the extraction of TMD information relevant for experiment from lattice calculations.

\section{Summary}

The present study focused on the Boer-Mulders shift in a pion, with a particular emphasis on obtaining information concerning the behavior of this type of TMD observable for large CollinsSoper parameter $\hat{\zeta}$. The Boer-Mulders shift is determined from pion matrix elements of a quark bilocal operator containing a staple-shaped gauge link which serves to incorporate final/initial state effects (for SIDIS/DY); the connection between the Lorentz frame preferred for the lattice calculation (in which the staple is defined at a single Euclidean time) and the Lorentz frame preferred for the phenomenological definition of TMDs (in which the staple direction approaches the light cone from the space-like side, as parametrized by $\hat{\zeta}$ ) is achieved by extracting invariant amplitudes from the data. The results for the Boer-Mulders shift suggest an early onset of asymptotic behavior as a function of $\hat{\zeta}$; conclusions about the light-cone limit thus appear to be within reach of lattice calculations. Furthermore, the Boer-Mulders shift for $u$-quarks in protons, investigated previously in [9], and $\pi^{+}$mesons was observed to be quantitatively similar.

\section{Acknowledgments}

The lattice calculations performed in this work relied on the Chroma software suite [12] and employed computing resources provided by the U.S. DOE through USQCD at Jefferson Lab. Support by the Heisenberg-Fellowship program of the DFG (P.H.), SFB/TRR-55 (A.S.), and the U.S. DOE through grants DE-FG02-96ER40965 (M.E.) and DE-FG02-94ER40818 (J.N.), as well as through contract DE-AC05-06OR23177, under which Jefferson Science Associates, LLC, operates Jefferson Laboratory (B.M.), is acknowledged.

\section{References}

[1] D. Boer, M. Diehl, R. Milner, R. Venugopalan, W. Vogelsang et al., arXiv:1108.1713.

[2] T. C. Rogers and P. J. Mulders, Phys. Rev. D 81, 094006 (2010).

[3] S. M. Aybat and T. C. Rogers, Phys. Rev. D 83, 114042 (2011).

[4] J. C. Collins, Foundations of Perturbative QCD (Cambridge University Press, 2011).

[5] S. M. Aybat, J. C. Collins, J.-W. Qiu and T. C. Rogers, Phys. Rev. D 85, 034043 (2012).

[6] M. G. A. Buffing and P. J. Mulders, arXiv:1309.4681.

[7] A. Airapetian et al., HERMES Collaboration, Phys. Rev. Lett. 103, 152002 (2009).

[8] J. Collins and A. Metz, Phys. Rev. Lett. 93, 252001 (2004).

[9] B. Musch, P. Hägler, M. Engelhardt, J. W. Negele and A. Schäfer, Phys. Rev. D 85, 094510 (2012).

[10] C. Aubin, C. Bernard, C. DeTar, J. Osborn, S. Gottlieb, E. Gregory, D. Toussaint, U. Heller, J. Hetrick and R. Sugar, Phys. Rev. D 70, 094505 (2004).

[11] M. Burkardt and B. Hannafious, Phys. Lett. B658, 130 (2008).

[12] R. G. Edwards and B. Joó, SciDAC Collaboration, Nucl. Phys. Proc. Suppl. 140, 832 (2005). 